\title{
SNOMED CT: Who Needs to Know What?
}

\author{
Ed Conley ${ }^{1,2}$, Tim Benson ${ }^{1,3}$ \\ ${ }^{1}$ Wellcome Trust Sintero Server Project, Cardiff University School of Computer Science \& Informatics, UK \\ ${ }^{2}$ Severnside Translational Research Alliance (SARTRE) at Cardiff and Bristol Universities, UK \\ ${ }^{3}$ Abies Ltd, Hermitage, Berkshire, UK
}

\begin{abstract}
Adoption of SNOMED CT has not been as quick and easy as many people had hoped or expected. One reason is lack of education and hence understanding of what SNOMED CT does and how it works. We set out to answer the question "who needs to know what?" about SNOMED CT to help establish priorities for UK higher education. We devised an online questionnaire and obtained 177 responses, $51 \%$ health IT professionals, $42 \%$ clinicians. The sample was self-selecting of those with knowledge of SNOMED CT. The level of reported competence was greater among health IT professionals (33\% rated themselves as competent) than among clinicians ( $5 \%$ rated themselves as com-
\end{abstract}

petent) $92 \%$ of those who felt competent had received 3 or more days of training in SNOMED CT. This indicates the need for formal training in SNOMED CT. Most respondents indicated that health IT professionals ought to have a high level of competence in SNOMED CT, such that they are able to explain most if not all aspects of SNOMED CT to others. On the other hand, clinicians only require a fairly basic understanding.

\section{Keywords}

SNOMED CT, Medical Informatics, Education

\section{Correspondence to:}

Tim Benson

Abies Ltd

Address: 14 Pinewood Crescent, Hermitage,

Thatcham, Berkshire RG18 9WL, UK

E-mail: tim.benson@abies.co.uk
EJBI 2011; 7(2):40-47

received: June 20, 2011

accepted: September 29, 2011

published: December 23, 2011

\section{Introduction}

SNOMED CT is an acronym for Systematised Nomenclature of Medicine Clinical Terms. SNOMED CT is owned and managed by the International Health Terminology Standards Development Organisation (IHTSDO), based in Copenhagen, and is funded by direct contributions from governments. In 2011 these include: Australia, Canada, Cyprus, Denmark, Estonia, Lithuania, New Zealand, Singapore, Slovak Republic, Slovenia, Spain, Sweden, The Netherlands, United Kingdom and United States.

SNOMED CT has been given a central role as a core component of information technology strategy in many leading countries. For example, SNOMED CT is one of the coding schemes mandated in the US government's incentive program for meaningful use of electronic health records [1. In the UK, the Department of Health has confirmed that it intends for SNOMED CT to be the sole supported terminology from 1st April 2015 [2].

Given its importance, it is reasonable to expect a high level of knowledge and understanding of it within the health informatics community. However, anecdotal evidence suggests that this is not the case.

Formally, SNOMED CT is a logic-based representation of meanings, organised in a directed acyclic graph. However, most people who use SNOMED CT do not understand the previous sentence, which demonstrates the problem.

We report a survey, which was designed to clarify two questions: how much do interested clinicians and health IT professionals already know about SNOMED CT, and who needs to know what.

SNOMED CT is a large, comprehensive, multilingual clinical terminology for use in computer systems. It comprises over 291,000 active clinical concepts, 758,000 English descriptions and 923,000 defining relationships (IHTSDO, 2010). By comparison, the main part of the International Classification of Diseases (ICD-10) has 10,760 items (excluding external causes). The sheer scale of SNOMED CT is one of the major challenges in using it.

SNOMED CT was developed by the College of American Pathology by merging its own SNOMED RT (Reference Terminology) with the NHS Clinical Terms Version 3 (CTV3), also known as the Read Codes Version 3. This 
work began in 1999 and the first release was on 31 January 2002.

The primary reason for developing SNOMED CT was the recognition that previous coding schemes, based on hierarchical taxonomic principles (tree-structures) were not 'fit-for-purpose'. Problems arising from older terminologies escalate over the years and it can be surprisingly hard to migrate from one version to another. The designers of SNOMED CT sought to address these problems and meet twelve specific requirements of a health terminology, which were summarised by Cimino 3 .

1. Concept orientation: The idea of a concept with a single clinical meaning is the central tenet. Each concept has a single code or identifier and may be described using several human-readable terms which have the same meaning (synonyms) in one or more languages.

2. Content: Clinical terminologies need to be comprehensive in the breadth of the concepts included and the terms used to describe them and in the level of detail used. They require a robust means to incorporate changes (additions and deletions) and translation into other languages.

3. Concept permanence: Once a concept has been created it is persistent. It never disappears or changes its meaning.

4. Non-semantic identifiers: Semantic information is an attribute of a concept, not part of the identifier or code.

5. Polyhierarchy: Clinical concepts are naturally multi dimensional and can have multiple parents.

6. Formal definitions: Concepts in SNOMED CT are defined using a formal language expressing explicit computable relationships with other concepts.

7. Rejection of NEC terms: Catch-all categories such as "not elsewhere classified (NEC)" and "not otherwise specified (NOS)" are not used. This is because the meaning of an NEC class changes if a new category is added which includes some of its meaning.

8. Multiple granularity: Different types of user (clinicians, managers, policy makers) typically need different levels of information granularity.

9. Multiple consistent views: The coding scheme is viewable in different but consistent ways, so each user need only see what is relevant to them.

10. Context representation: Context (such as who made a statement, when and where) is an important aspect of medical data.

11. Graceful evolution: Terminologies need to change as knowledge develops, so the system evolves in a way that legacy data is preserved.
12. Recognition of redundancy: As systems change, concepts become redundant, but are not entirely deleted.

SNOMED CT is architecturally a substantial step forward over all previous systems with comparable scope. Its underlying structure means that changes can be incorporated relatively easily as the system evolves.

\section{Scope of study}

The need for more education and training in SNOMED CT is widely recognised, but many educators are unsure about what health IT professionals and clinical users of IT systems need to know. Little is known about the health informatics community's current level of knowledge, their expectations and perceived learning needs about SNOMED CT.

We set out to illuminate these questions, focusing on two broad groups - health IT professionals and clinical end users, mainly but not exclusively on the UK.

\section{Method}

We used an on-line survey tool, which permitted a mix of response types and an opportunity to test broad concept recognition patterns and elicit free ideas.

We used our own knowledge of SNOMED CT to make list a range of topics that could be relevant or interesting to the two broad survey groups of clinicians and health IT professionals. We only asked questions that we were genuinely interested to know how people would answer. The questions were worded in a way that we could not confidently predict the answer. The questionnaire was developed in a number of interactive sessions between the two authors; the beta version was piloted on ten subjects with varying knowledge of SNOMED CT.

The final version of the questionnaire was deployed using the Survey Monkey on-line questionnaire system, which also provides useful analysis facilities. We sent a single email invitation to several hundred people in the health informatics communities in the UK and internationally, including past attendees at SNOMED and HL7 conferences and training courses in the UK, to members of the BCS Primary Healthcare Specialist Group and the NHS Clinical Leaders network. The invitation directed recipients to the survey site. No reminders were sent.

The results were analysed primarily using the online tools provided by Survey Monkey.

\section{Results}

\subsection{Respondent's Profile}

177 respondents started the survey with $136(76.8 \%)$ completing it. $20 \%$ of responses came from outside the UK, mainly health IT professionals. 
Table 1] shows a balance between health IT professionals and other user roles. Respondents were able to select more than one role.

Table 1: Principal respondent job role.

\begin{tabular}{|l|c|}
\hline Respondent role & All respondents \\
\hline \hline Health IT Professional & $82(50.9 \%)$ \\
\hline Clinician & $67(41.6 \%)$ \\
\hline Manager & $12(7.5 \%)$ \\
\hline Other & $18(11.2 \%)$ \\
\hline
\end{tabular}

Respondents had a wide range of academic qualifications across different subject areas, which illustrates the multi-disciplinary nature of health informatics. The mean number of academic qualifications reported per respondent was 1.53 (Table 2).

Table 2: Respondents' academic qualifications.

\begin{tabular}{|l|c|}
\hline Academic qualification & All respondents \\
\hline \hline Medicine & $69(41.6 \%)$ \\
\hline IT/ computing / systems science & $44(26.5 \%)$ \\
\hline Health Informatics & $30(18.1 \%)$ \\
\hline Other clinical (e.g. nursing) & $29(17.4 \%)$ \\
\hline Other technical (e.g. engineering) & $38(22.9 \%)$ \\
\hline Other academic qualifications & $45(26.9 \%)$ \\
\hline Qualifications/Respondents & $\mathbf{2 5 5 / 1 6 7 ( 1 . 5 3 )}$ \\
\hline
\end{tabular}

\subsection{Training and Education Experience}

The amount of SNOMED CT training received is shown in Table 3, 26.9\% of respondents had received 3 or more days of training, rather more than had received less than one day $(13.5 \%)$ or one or two days training $(15.2 \%)$.

This result was unexpected - we had expected a decrease in the numbers of respondents who had received more training. This is probably an artefact of this respondent population, which includes a high proportion of SNOMED experts.

Almost $50 \%$ of health IT professionals had received 3 or more days training in contrast to less than $8 \%$ of clinicians. Almost $70 \%$ of clinicians who responded had received no formal training in SNOMED CT, in contrast to $17 \%$ of the health IT professionals.

\subsection{Competence}

We found a strong relationship between respondent's self-reported knowledge/competence and the amount of formal training and education (in SNOMED CT) they had received (Table 4 ).

$26(17 \%)$ respondents rated themselves as competent, in comparison with $46(27 \%)$ who had received three or more days training. Of those who rated themselves as competent in SNOMED CT, $92 \%$ had received three or more days training and education.

Unexpectedly few people feel competent on all aspects of SNOMED CT, and an important conclusion is that training for 3 or more days is probably needed to deliver a perception of competence. In this context, most SNOMED CT training courses only take 1 or 2 days.

$28 \%$ of the respondents ( $74 \%$ of whom were clinicians) indicated that they knew nothing about SNOMED CT; the large majority of these answered "Don't Know" to most of the other questions. SNOMED CT is not "intuitively obvious"; it needs to be taught.

\subsection{Detailed Understanding of SNOMED CT}

We asked each respondent to say whether a series of eight statements about SNOMED CT were true or false or they did not know (Table 5). These statements were chosen to cover a range of subject areas, which might be covered in a clinical terminology course.

All of the statements are true. They are listed in order of the number of correct answers received. The number of "don't know" answers provides an indicator of which aspects of SNOMED CT are least well understood.

\subsection{Knowledge Needs for Clinical Users}

We asked which of these detailed aspects of SNOMED CT do clinicians need to know about (Table 6). The statements are listed in order of importance for clinicians to understand.

Most respondents (55\%) reported that clinical users need to understand that SNOMED CT is important for information reuse; one third, that SNOMED CT concepts can have more than one parent in their hierarchy; others aspects had substantially lower scores, although all received some support.

These results support a conclusion that clinicians need to be taught a small number of basic principles about SNOMED CT.

\subsection{Knowledge Needs for Health IT Professionals}

We asked if health IT professionals should be able to explain each of these aspects of SNOMED CT (Table 7). Statements are listed in order of importance for health IT professionals to be able to explain.

The majority thought that health IT professionals should be able to explain most of these aspects of SNOMED CT. This is an important finding because most higher education courses in health informatics do not currently teach clinical terminology and SNOMED at the level that this implies.

These responses suggest that health IT professionals should be taught quite a lot about clinical terminology 
Table 3: Amount of formal training and education in SNOMED CT.

\begin{tabular}{|l|c|c|c|}
\hline Formal SNOMED CT Training Days & Clinician & Health IT Professional & All respondents \\
\hline \hline None & $46(69.7 \%)$ & $14(17.1 \%)$ & $76(44.4 \%)$ \\
\hline Less than one day & $10(15.2 \%)$ & $9(11.0 \%)$ & $23(13.5 \%)$ \\
\hline One or two days & $5(7.6 \%)$ & $19(23.2 \%)$ & $26(15.2 \%)$ \\
\hline Three or more days & $5(7.6 \%)$ & $40(48.8 \%)$ & $46(26.9 \%)$ \\
\hline \hline TOTAL & $66(100 \%)$ & $82(100 \%)$ & $171(100 \%)$ \\
\hline
\end{tabular}

Table 4: Respondents' knowledge about SNOMED CT in relation to the amount of training received.

\begin{tabular}{|l|c|c|}
\hline Knowledgeable about SNOMED CT & All respondents & 3 or more days training \\
\hline \hline Competent & $26(16.7 \%)$ & $24(92.3 \%)$ \\
\hline Intermediate & $33(21.4 \%)$ & $13(39.4 \%)$ \\
\hline Novice & $54(34.6 \%)$ & $4(7.4 \%)$ \\
\hline Know nothing & $43(27.9 \%)$ & $0(0 \%)$ \\
\hline \hline TOTAL & 156 & $41(26.3 \%)$ \\
\hline
\end{tabular}

Table 5: Respondents' knowledge of detailed aspects of SNOMED CT.

\begin{tabular}{|c|c|c|}
\hline Aspect of SNOMED CT & True & Don't Know \\
\hline SNOMED CT is important for information reuse & $102(66.2 \%)$ & $46(29.9 \%)$ \\
\hline SNOMED CT concepts can have more than one parent in their hierarchy & $90(58.4 \%)$ & $55(35.7 \%)$ \\
\hline $\begin{array}{l}\text { SNOMED CT concepts may be defined by their relationships with other con- } \\
\text { cepts }\end{array}$ & $87(56.5 \%)$ & $62(40.3 \%)$ \\
\hline $\begin{array}{l}\text { SNOMED CT is represented using either pre-coordinated concepts or post- } \\
\text { coordinated expressions }\end{array}$ & $72(47.1 \%)$ & $75(49.0 \%)$ \\
\hline The SNOMED CT concept model constrains what relationships are allowed & $63(41.2 \%)$ & $79(51.6 \%)$ \\
\hline SNOMED CT uses variable length numeric codes & $60(39.2 \%)$ & $66(43.5 \%)$ \\
\hline SNOMED CT Concepts are either fully defined or are marked as primitive & $54(35.1 \%)$ & $94(61.0 \%)$ \\
\hline Subsumption-testing tests if one node is a child of another & $43(27.9 \%)$ & $109(70.8 \%)$ \\
\hline
\end{tabular}

including Description Logic, post-coordination and subsumption testing.

\subsection{Why Learn SNOMED CT?}

People need to be motivated to learn SNOMED CT. So, we asked about six possible reasons to learn it (Table 8). The results are shown in order of importance.

The most widely chosen reason for learning SNOMED $\mathrm{CT}$ was that it is an officially recognised standard. Government support for SNOMED CT is clearly a key factor.

Less than one third of the respondents said that helping their career was a very important reason for learning SNOMED. These results indicate that the incentives for individuals to learn SNOMED CT are not yet very strong.

\subsection{Benefits of SNOMED CT}

We asked whether SNOMED CT would be important in standardising a number of subject areas in health informatics (Table 9).
Almost two-thirds of respondents said that SNOMED CT will be "very important" in standardising interoperability (65\%), data collection (65\%) and clinical decision support $(62 \%)$.

This positive view of the benefits of SNOMED CT, in contrast with the smaller number of people (30.4\%) who said that learning SNOMED CT would be very important in helping their career, suggests that some respondents felt that the benefits of SNOMED CT are more likely to accrue to others, not necessarily themselves.

\subsection{Barriers to Adoption}

We know that the uptake of SNOMED CT has been slow, so we listed several of the most obvious barriers to the adoption of SNOMED CT, and asked respondents how strongly they agreed (Table 10p. The strongest response was "I totally agree".

Our respondents rated the cost of training and educating staff in SNOMED CT to be the biggest barrier (59.4\%), followed by the inherent complexity of SNOMED 
Table 6: Do clinical users of the electronic health record need to understand the following aspects of SNOMED CT?

\begin{tabular}{|l|c|c|}
\hline Aspect of SNOMED CT (Clinical users) & $\begin{array}{l}\text { Definitely need to un- } \\
\text { derstand }\end{array}$ & Not at all \\
\hline \hline SNOMED CT is important for information reuse & $83(55.0 \%)$ & $6(4.0 \%)$ \\
\hline $\begin{array}{l}\text { SNOMED CT concepts can have more than one parent in their } \\
\text { hierarchy }\end{array}$ & $50(33.3 \%)$ & $20(13.3 \%)$ \\
\hline $\begin{array}{l}\text { SNOMED CT concepts may be defined by their relationships with } \\
\text { other concepts }\end{array}$ & $34(22.7 \%)$ & $19(12.7 \%)$ \\
\hline $\begin{array}{l}\text { SNOMED CT is represented using either pre-coordinated con- } \\
\text { epts or post-coordinated expressions }\end{array}$ & $25(16.8 \%)$ & $26(17.4 \%)$ \\
\hline $\begin{array}{l}\text { The SNOMED CT concept model constrains what relationships } \\
\text { are allowed }\end{array}$ & $17(11.4 \%)$ & $36(24.2 \%)$ \\
\hline $\begin{array}{l}\text { SNOMED CT Concepts are either fully defined or are marked as } \\
\text { primitive }\end{array}$ & $7(7.4 \%)$ & $63(42.3 \%)$ \\
\hline SNOMED CT uses variable length numeric codes & $6(4.1 \%)$ & $44(29.7 \%)$ \\
\hline Subsumption-testing tests if one node is a child of another & & \\
\hline
\end{tabular}

Table 7: Should Health IT professionals (e.g. at Masters level) be able to explain the following aspects of SNOMED CT?

\begin{tabular}{|l|c|c|}
\hline Aspect of SNOMED CT (Health IT Professionals) & $\begin{array}{l}\text { Definitely need to be } \\
\text { able to explain }\end{array}$ & Not at all \\
\hline \hline SNOMED CT is important for information reuse & $98(68.1 \%)$ & $2(1.4 \%)$ \\
\hline $\begin{array}{l}\text { SNOMED CT concepts can have more than one parent in their } \\
\text { hierarchy }\end{array}$ & $93(65.5 \%)$ & $2(1.4 \%)$ \\
\hline $\begin{array}{l}\text { SNOMED CT concepts may be defined by their relationships with } \\
\text { other concepts }\end{array}$ & $87(60.8 \%)$ & $3(2.1 \%)$ \\
\hline $\begin{array}{l}\text { SNOMED CT is represented using either pre-coordinated con- } \\
\text { epts or post-coordinated expressions }\end{array}$ & $82(57.3 \%)$ & $2(1.4 \%)$ \\
\hline $\begin{array}{l}\text { The SNOMED CT concept model constrains what relationships } \\
\text { are allowed }\end{array}$ & $82(57.3 \%)$ & $6(2.1 \%)$ \\
\hline SNOMED CT uses variable length numeric codes & $76(53.1 \%)$ & $3(2.1 \%)$ \\
\hline $\begin{array}{l}\text { SNOMED CT Concepts are either fully defined or are marked as } \\
\text { primitive }\end{array}$ & $74(51.7 \%)$ & $4(2.8 \%)$ \\
\hline Subsumption-testing tests if one node is a child of another & $68(47.6 \%)$ & \\
\hline
\end{tabular}

Table 8: Reasons for learning SNOMED CT.

\begin{tabular}{|l|c|}
\hline REASONS for learning SNOMED CT & Answered "Very Important" \\
\hline \hline $\begin{array}{l}\text { SNOMED CT is the official standard coding scheme for exchanging cli- } \\
\text { nical information in the NHS }\end{array}$ & $77(58.3 \%)$ \\
\hline SNOMED CT is used internationally & $76(57.1 \%)$ \\
\hline SNOMED CT is one of the cornerstones of Health Informatics & $66(50.0 \%)$ \\
\hline $\begin{array}{l}\text { SNOMED CT is comprehensive in a way that other coding schemes are } \\
\text { not }\end{array}$ & $60(46.2 \%)$ \\
\hline Knowing SNOMED CT will help my career & $41(30.4 \%)$ \\
\hline We are already using SNOMED CT in house & $34(26.4 \%)$ \\
\hline
\end{tabular}

CT $(57.5 \%)$ and the lack of good training and education materials $(53.7 \%)$.

Technical issues such as post-coordination, data migration and finding the right code were also seen as significant barriers to adoption.
This clearly demonstrates that for this sample at least, the biggest barriers to the adoption of SNOMED CT can only be overcome by a greatly increased emphasis on education and training. 
Table 9: Do you think that SNOMED CT will help in standardising.

\begin{tabular}{|l|c|}
\hline Aspects for standardising & Answered "very important" \\
\hline \hline Interoperability & $87(64.9 \%)$ \\
\hline Data collection & $88(64.7 \%)$ \\
\hline Clinical decision support & $84(62.2 \%)$ \\
\hline Analysis and research & $80(58.8 \%)$ \\
\hline Retrieval and display of patient records & $71(53.0 \%)$ \\
\hline Health service management & $60(44.1 \%)$ \\
\hline
\end{tabular}

Table 10: Barriers to the adoption of SNOMED CT.

\begin{tabular}{|l|c|}
\hline Barriers to the adoption of SNOMED CT? & I totally agree \\
\hline \hline Cost and time needed to train staff & $79(59.4 \%)$ \\
\hline SNOMED CT is inherently complex & $77(57.5 \%)$ \\
\hline There is a lack of good training and education materials & $73(53.7 \%)$ \\
\hline Post-coordination adds another layer of complexity & $64(47.8 \%)$ \\
\hline Migration of legacy codes & $63(46.7 \%)$ \\
\hline It can be difficult to find the right code & $54(40.0 \%)$ \\
\hline The documentation is too long and complex & $47(35.1 \%)$ \\
\hline Difficult to add your own local codes & $30(22.4 \%)$ \\
\hline
\end{tabular}

Table 11: Actual and desired ways of learning about SNOMED CT.

\begin{tabular}{|l|c|c|}
\hline How did you learn about SNOMED CT? & How I learnt & Best ways to learn \\
\hline \hline Pick it up from colleagues & $71(55.0 \%)$ & $89(70.6 \%)$ \\
\hline Read user guides and books & $69(53.1 \%)$ & $77(62.1 \%)$ \\
\hline Face-to-face tuition/presentations & $65(50.4 \%)$ & $121(92.4 \%)$ \\
\hline Learning by doing (e.g. practical examples) & $58(45.7 \%)$ & $125(96.2 \%)$ \\
\hline Watching video / web presentations & $22(17.5 \%)$ & $91(74.0 \%)$ \\
\hline
\end{tabular}

Table 12: The most important aspects of SNOMED CT for the higher education curriculum.

\begin{tabular}{|l|c|}
\hline Important aspects of SNOMED CT for higher education curriculum & High priority \\
\hline \hline Understanding how SNOMED CT works & $85(64.9 \%)$ \\
\hline The strategic value of SNOMED CT & $81(62.3 \%)$ \\
\hline Choosing the right code & $70(54.7 \%)$ \\
\hline Use in interoperability & $69(53.5 \%)$ \\
\hline The SNOMED CT concept model & $67(52.8 \%)$ \\
\hline Analysis and reporting & $59(46.5 \%)$ \\
\hline Mapping to / from other code systems & $54(42.2 \%)$ \\
\hline Binding with information models & $49(38.9 \%)$ \\
\hline Post-coordinated expressions & $30(24.0 \%)$ \\
\hline Building sub-sets (ref-sets) & $24(18.8 \%)$ \\
\hline
\end{tabular}

\subsection{Mechanisms for Learning about SNOMED CT}

We asked how respondents had learnt SNOMED CT and how they thought people should learn (Table 11).

We found a difference between how people had learnt for themselves - picking it up from colleagues (55\%), reading $(53 \%)$ and face-to-face tuition (50\%) - and their views of the best way to learn - learning by doing (e.g. practical examples) (96\%), face to face tuition (92\%) and watching video / web presentations (74\%), although all methods had substantial support.

The survey confirms that people use a mix of learning strategies and one method will not suit everyone. These results suggest that e-learning (video/web presentations) should be part of the solution, but is not whole. 


\subsection{Curriculum Development}

We asked respondents to select from a list, those items of highest priority for curriculum development (Table 12). The top priorities were understanding how SNOMED CT works $(65 \%)$ and the strategic value of SNOMED CT $(62 \%)$.

Fewer votes were given to more advanced topics such as post-coordination and building sub-sets (ref-sets). However a vote of 30 respondents (24\%) saying that "postcoordinated expressions" should be "high priority" is a significant minority view.

\section{Discussion}

The main conclusion of this survey is that health IT professionals need to gain a substantially higher level of knowledge and expertise in SNOMED CT than most have today. There is a substantial gap between what health IT professionals need to know about SNOMED CT and what is taught in most universities and college courses on health informatics. In particular, we recommend that all health informatics professionals should learn Description Logic, which forms the basis of the way that SNOMED CT concepts are defined and post-coordinated expressions are put together.

There is a lack of adequate educational material on SNOMED CT which is fit for this purpose. Nine years after SNOMED CT was first released, not one book has yet been published that is devoted to it.

The Australian National e-Health Transition Authority (NEHTA) has published on YouTube two excellent video tutorials by Kent Spackman [4] with accompanying powerpoint sets [5]. However the total length is only about two and a half hours.

We have to educate the educators, but we lack sufficient well-qualified senior lecturers to motivate and educate their peers.

It is about thirty years since Blois 6 showed that clinical terminology is the key distinguishing feature of health IT, being central to medical thought and uniquely broad and deep. Clinical terminology is a core competence of health informatics. However, those who develop curricula for health informatics have not placed adequate emphasis on clinical terminology.

For example, the American Medical Informatics Association (AMIA) White Paper on Core Content for the Subspecialty of Clinical Informatics published in 2009 [7] lists clinical terminology as just one of 186 subject items. In 2010 IMIA revised its recommendations on education in health informatics [8], which includes references to 37 major publications on health informatics competencies. The new IMIA recommendations say "particular emphasis should be given to information literacy, health terminology, coding and classification systems, the electronic health record, and evaluation methodology", but only one item (1.17 Nomenclatures, vocabularies, terminologies, ontologies and taxonomies in BMHI) out of 48 listed knowledge/skill domains is explicitly devoted to clinical terminology.

Clinicians need a basic understanding of clinical terminology and SNOMED CT in particular but this need not include the technical details.

Access to SNOMED CT needs to be made easier. The whole of SNOMED CT should be made transparently accessible for inspection and review on the Internet. There are several adequate web-based browsers, but none support all of the facilities of SNOMED CT and each would benefit from further investment to make them more suitable as an educational resource.

Cooke 9] has suggested the following hierarchy of need to know:

- System designers and architects need to know everything!

- System configuration staff, information analysts and support staff need to understand the technical architecture and subsets/refsets.

- Trainers need to understand how searches work, but do not need to understand SNOMED CT structure.

- Clinicians need to know how to do searches, but no technical details of SNOMED CT.

This hierarchy is confirmed by this survey. Most respondents indicated that health IT professionals should have a high level of competence in SNOMED CT, such that they are able to explain most if not all aspects of SNOMED CT to others. On the other hand, clinicians only require a fairly basic understanding of clinical terminology. This survey is complementary to other surveys which focus on the completeness, accuracy and use of SNOMED CT itself [10].

Cornet and de Keizer analysed some 250 papers published between 1966 and 2006 related to the use of SNOMED in all its forms 11. The two largest groups of papers concerned (1) comparisons of SNOMED with other terminologies and (2) those in which a theory such as automated coding, natural language processing or description logic is illustrated using SNOMED as an exemplar. They found little in the literature about the use of SNOMED in clinical practice. Educational challenges were not mentioned.

This study is necessarily limited to the respondents who chose to reply, and the responses were biased towards people who were already knowledgeable about SNOMED CT.

\section{Conflict of Interest}

Tim Benson's company (Abies Ltd) provides training courses in SNOMED CT. 


\section{Acknowledgements}

We wish to thank all respondents for their time; the Department of Health Informatics Directorate for sponsorship of the work; Denise Downs Data Standards Implementation and Education Lead at Department of Health Informatics Directorate) for advice and support; Katherine Christensen (Knowledge Communities Implementation Manager, Informatics Development DH Informatics Policy and Planning Directorate) for assistance with distribution of survey materials and John Gutai, Chief Technical Architect at the International Health Terminology Standards Development Organisation (IHTSDO) for advice.

\section{References}

[1] US Department of Health and Human Services. Health Information Technology: Initial Set of Standards, Implementation Specifications, and Certification Criteria for Electronic Health Record Technology: Final Rule. 45 CFR Part 170 Federal Register, July 28, 2010.

[2] Jones P. The UK Edition of SNOMED CT as the Fundamental Standard for Clinical Terminology within the NHS in England. Department of Health Informatics Directorate, 2011.
[3] Cimino J. Desiderata for controlled medical vocabularies in the twenty-first century. Methods Inf Med, 1998. 37(4-5): p. 394-403.

[4] YouTube: http://www.youtube.com/watch?v=ISfoMR4aygc and http://www.youtube.com/watch?v $=\mathrm{CrZ46eXunzo}$

[5] IHTSDO: http://www.ihtsdo.org/publications/educationtraining.

[6] Blois M. Information and medicine: the nature of medical descriptions. 1984, Berkeley: University of California Press.

[7] Gardner R, et al. Core content for the subspecialty of clinical informatics. J Am Med Inform Assoc, 2009. 16(2): p. 153-7.

[8] Mantas J, et al. Recommendations of the International Medical Informatics Association (IMIA on Education in Biomedical and Health Informatics, First Revision. Methods Inf Med 2010;49:105-120 doi:10.3414/ME5119.

[9] Cooke M. Implementing SNOMED CT; Practicalities and Challenges. IHTSDO, 2009. http://www.ihtsdo.org /fileadmin/user_upload/Docs_01/Recourses/Implementing SNOMED $\mathrm{C} \overline{\mathrm{T}} /$ Implementing SNOMED CT Practicali-

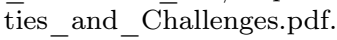

[10] Elhanan G, Perl Y, Geller J (2010) A survey of direct users and uses of SNOMED CT: 2010 Status. AMIA 2010 Symposium Proceedings 207-211. http://1.usa.gov/nFTxX7

[11] Cornet R, de Keizer N. Forty years of SNOMED: a literature review. BMC Medical Informatics and Decision Making 2008, 8(Suppl 1):S2 doi:10.1186/1472-6947-8-S1-S2 